\title{
Comparative Study of Information Security Risk Assessment Frameworks
}

Umesh Kumar Singh $^{\# 1}$, Chanchala Joshi ${ }^{\# 2}$

\#1 Institute of Computer Science, Vikram University, Ujjain, India \#2 Institute of Computer Science, Vikram University, Ujjain, India,

\section{ABSTRACT}

With the increasing need of securing organization's computing environment, a security risks management framework is essentially needed that define the security risks management process accurately. In this regard, numerous risks management frameworks have been developed, and many more are emerging every day. They all have very different perspectives and addressing problems differently, though with the same basic goal of risks mitigation in direction of information security. Information is a critical asset for every organization and hence development and implementation of strategic plans for information security risks mitigation should be an essential part of every organizations operation. This paper compares and analyzes the different activities, inputs and outputs required by each information security risk assessment models. The primary goal of the paper is to identify which information security risk assessment model assesses information security risk effectively. The comparative study helps in evaluating the models' applicability to an organization and their specific needs.

Key words: information security; vulnerability analysis; risks assessment model; risks management

\section{Corresponding Author: Chanchala Joshi}

\section{INTRODUCTION}

Vulnerability assessment is the examination of weaknesses that may be exploited by identified threats. This assessment might take into account the environment and existing safeguards. In organizations', the need for vulnerabilities detection and assessment is usually underestimated till now. It is just considered as a formality activity and use by very fewer people. By using regular and efficient vulnerability assessment, we can reduce the substantial amount of risk to be attacked and have more secure systems.

An information security risks management framework is a series of defined processes that are used to define strategies and procedures around the implementation and continuing management of information security controls in an enterprise system context. These frameworks are a "blueprint" for developing an information security program to manage risks and overcome vulnerabilities. Information security process can utilize these frameworks to define and prioritize the tasks required to build security into an organization. 
DOI: https://dx.doi.org/10.26808/rs.ca.i8v2.08

An information security risks management framework establishes the rules for what is assessed, who needs to be involved. It decides the criteria for quantifying, qualifying and comparing degrees of risk and produces the documentation as a result of assessments and follow-on activities. The goal of a framework is to establish an objective measurement of risk that will allow an organization to understand potential risk to critical information and assets both qualitatively and quantitatively [1]. A critical risk assessment framework defines security risk efficiently for an organization's computing environment. In the end, the risks management framework provides the plans to improve security level and recommend the countermeasures to bring the security risks to acceptable levels.

With the increase of the need to properly secure organization's computing environment, an increase in the number and diversity of frameworks has come to achieve this. In the past decade, many security risk assessment frameworks have been developed, and each has own procs and cons. Some frameworks were developed for specific organizations as well as different regulatory compliance goals. However, there is a significant overlap in general security concepts as each one evolves. This paper evaluates standard information security framework to designate the right Information Security Risk Assessment Framework.

The remainder of the paper is organized as follows. Section 2 designates the problem statement and research motivation behind the work. Section 3 provides some background discussion on what Information Systems are and on security challenges facing them. In section 4, we review standard information security frameworks OCTAVE, FAIR, NIST, COBIT and TARA. Section 5 provides a detailed analysis and comparison of the presented frameworks. We draw some concluding remarks in Section 6.

\section{RESEARCH MOTIVATION}

Cybercrooks revealed new levels of ambition in past few years by extraordinary attacks. Since the last few years, the total number of security incidents was on track to surpass the previous year. According to Symantec's 2016 Internet Threat Report [2], there is 125\% increase in targeted attacks from the year before in 2015. The results of recent investigations outputs have been the prime motivation for this research. In past few years, Vulnerability Assessment has become one of the most important research areas in information security. It is an emerging preventive information security technology which tests network security infrastructure and systems for known weaknesses. Vulnerability assessment techniques are evolving and facing many open issues. Till date, these problems have not been directed correctly. The existing methods do not "raise the bar" for the cyber-criminals, while their cost for the defender regarding resources that need to be devoted to detection can be significant.

Security breaches are increasingly reported; research and development team recently published that this year (in 2017) the total number of security incidents was on track to surpass 2016. The number of new vulnerabilities listed in National Vulnerability Database was 4,150 in 2011, 5286 in 2012, 5186 in 2013, 7937 in 2014, 6487 in 2015 and 6446 up to May 2017 [3]. Table 1 summarizes the CVSS severity distribution over last six years.

Table 1: CVSS severity distribution over time (2011 to May 2017) 
DOI: https://dx.doi.org/10.26808/rs.ca.i8v2.08 International Journal of Computer Application (2250-1797)

Issue 8 Volume 2, March-April 2018

\begin{tabular}{|l|l|l|l|l|}
\hline Year & Low & Medium & High & Total \\
\hline 2011 & 260 & 2068 & 1822 & 4150 \\
\hline 2012 & 509 & 3013 & 1764 & 5286 \\
\hline 2013 & 519 & 2930 & 1737 & 5186 \\
\hline 2014 & 662 & 5356 & 1919 & 7937 \\
\hline 2015 & 591 & 3489 & 2407 & 6487 \\
\hline 2016 & 619 & 3357 & 2470 & 6446 \\
\hline Up to May 2017 & 609 & 3886 & 1887 & 6382 \\
\hline
\end{tabular}

Fig.1 shows the CVSS severity distribution (High, Medium and Low) of vulnerabilities since 2001 to May 2017. As shown in the figure the number of vulnerabilities reported was 1677 in 2001 which increased to 6446 in 2016 . This shows approximately $385 \%$ increase in the rate of evolution of vulnerabilities in last few years. Moreover, this trend seems likely to continue, with a large and growing number of vulnerabilities discovered and published every year.

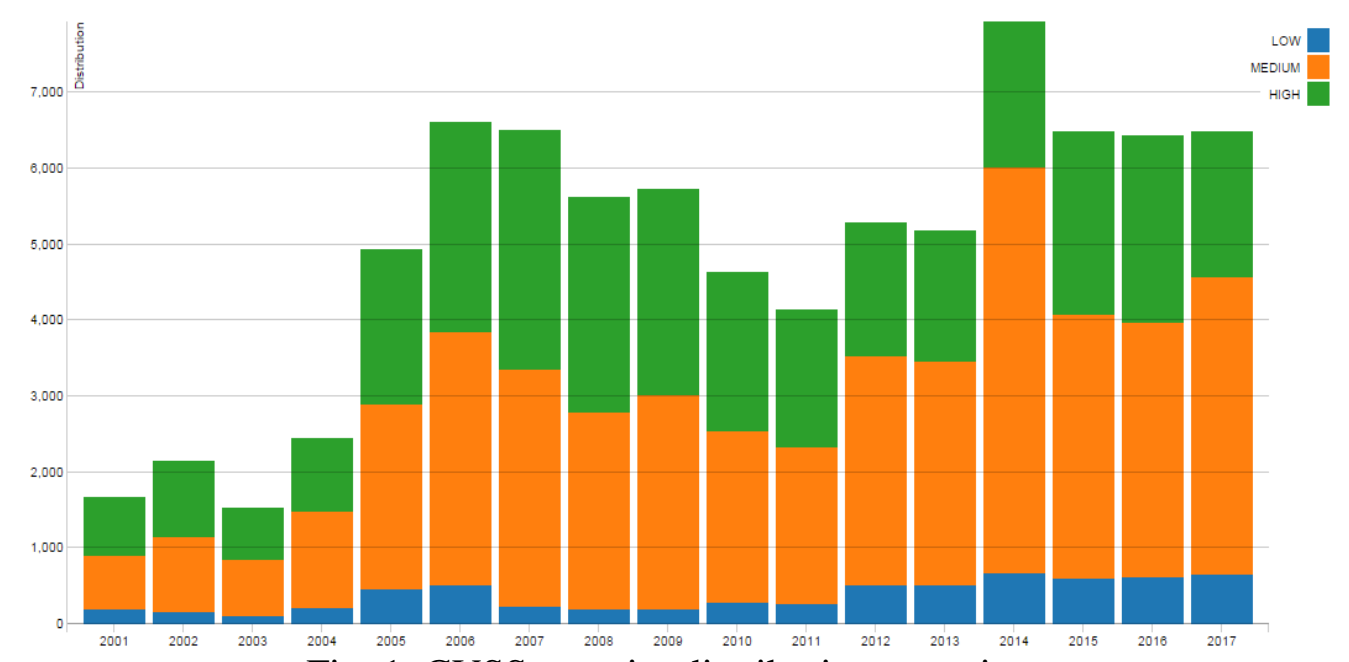

Fig. 1. CVSS severity distribution over time

With the advancement of Information Technology, computing and network applications have become an essential and integral part of universities environment. Today's all organizations are approaching towards the technological advancement. The greater access to technology results in the valuable operational environment, on the other hand, can also result in a vulnerable computing environment with more security threats. There are many risk assessment models. However, there is no mechanism to assist organizations in finding which one is the best to be employed for the organization. 
DOI: https://dx.doi.org/10.26808/rs.ca.i8v2.08

International Journal of Computer Application (2250-1797)

Issue 8 Volume 2, March-April 2018

\section{INFORMATION SECURITY}

Information security, sometimes shortened to InfoSec is an approach or method of preventing unauthorized access, use, disclosure, disruption, modification, inspection, recording or destruction of information [4]. Information security's primary focus is the balanced protection of the confidentiality, integrity and availability of data while maintaining a focus on efficient policy implementation, all without hampering organization productivity [5]. This is largely achieved through a multi-step risk management process that identifies assets, threat sources, vulnerabilities, potential impacts, and possible controls, followed by assessment of the effectiveness of the risk management plan

The prime of the information security risk assessment frameworks is an objective of assessing organization's network. To achieve this goal a recursive mechanism is applied that along with gathering inputs concerning vulnerabilities and threats, estimates the quantitative risk value that can be measured and treated.

Prevailing steps of information security risk assessment frameworks are,

$>$ Identifying and recognizing assets and stakeholders,

$>$ Identifying security requirements,

$>$ Evaluating vulnerabilities,

$>$ Investigating the effectiveness of controls,

$>$ Evaluation of risks by estimating frequency and impact of the exploit,

$>$ Designing remediation plans, and

$>$ Finally, drive decisions using powerful reporting.

\section{PROMINENT INFORMATION SECURITY RISKS ASSESSMENT FRAMEWORKS}

There are several models and methods with different approaches that help in the risk assessment process. This study will address the methods that support the risk assessment process and those which can be applied to information security. This section provides an overview of those methods, and a comparison that evaluates those different methodologies. We evaluate standard information security framework OCTAVE, FAIR, NIST, COBIT and TARA to designate the right Information Security Risk Assessment Framework.

\section{OCTAVE}

Operationally Critical Threat, Asset, and Vulnerability Evaluation generally abbreviated as OCTAVE is the standard security framework for measuring risk level and planning defenses against cyber assaults [6]. The framework describes a methodology to assist organizations to depreciate exposure to possible threats, determine the permissible consequences of attacks and deal with attacks that succeed. It is the most well-known of the risk frameworks which comes in three sizes [7].

The initial, full-featured version is a heavyweight process intended for large organizations with substantial documentation. One another version is designed for smaller organizations, OCTAVE-S. It is designed for organizations where the multi-disciplinary group may consist of the fewer people, and they are mainly technical communities with knowledge of the trade. InOCTAVE-S, the documentation load is low and the process is lightweight. The newest and 
advanced product in the OCTAVE group is Allegro, which takes a more focused approach than its predecessors along with the more of a lightweight feel. In, Allegro the assets are the information, demanding further training at the start of the process, and views applications, systems, and environments as containers. The extent of the assessment needs to be based on the information absorption, and identify and assess risks across the containers in which the information is stored, transmitted, or processed.

OCTAVE is constructed to leverage the expertise and experience of people of the organization. The first step is to build threats profiles based on the corresponding risk that an organization may pretend. The process goes on to carry a vulnerability assessment that is specific to a particular organization. One of the advantages of the OCTAVE series is that each of the frameworks exhibits patterns for worksheets to document each round of the process. These can either be applied directly or customized for a specific organization.

OCTAVE describes assets as including hardware, software, information, people, and systems. One of the significant drawbacks of OCTAVATE is its complexity, and it does not allow organizations to quantitatively model risk.

\section{FAIR}

FAIR abbreviation of Factor Analysis of Information Risk, is a risk management framework which is developed by Jack A. Jones to help organizations in understanding, analyzing, and measuring information risks [8]. It is a risk assessment framework for understanding, measuring and analyzing information risk for enabling well-informed decision making. FAIR is established to approach the weaknesses of security concern. The framework enables organizations for normalizing the risk, applying risks assessment, viewing overall organizational risks It assists in defending risk determination using advanced analysis and understanding how money and time will affect the organization's security. The main deficiency of FAIR is the lack of information about methodology and examples of how the methodology is applied.

\section{NIST}

NIST RMF (National Institute of Standards and Technology Risk Management Framework) is a general one that can be applied to any asset [9]. It covers a series of activities related to managing organizational risk, uses slightly different terminology than OCTAVE, but follows a similar structure. It does not present the capital of forms that OCTAVE does, but is relatively candid to follow. Its concision and focus on more physical components (e.g., systems) makes it a good nominee for organizations new to risk assessment. Moreover, because NIST defines it, it is approved for use by government agencies and organizations that work with them.

However, NIST is not available with computer support, where the templates could be used electronically, and reports could be created automatically for the vast number of data collected during the information risk assessment. It is used more as a guideline instead of a methodology.

\section{TARA}

TARA (Threat Agent Risk Assessment), the risk assessment framework is developed by Intel to helps organizations in managing risks by extracting the possible information about security attacks [10]. The TARA methodology recognizes which threats posture the highest risk, what 
they want to achieve and the possible methods they will use. The techniques are crossreferenced with existing vulnerabilities and instruments to discover most exhibited areas. The security strategy then focuses on these areas to minimize efforts while maximizing the effect. Intel states awareness of the most exposed areas allows the company to make better judgments about how to manage risks, which helps with adjusting spending, preventing impacts and leading to an acceptable level of residual risks [11]. The TARA methodology is outlined to be readily adapted when a company faces changes in threats, computing environments, behaviors or vulnerabilities. The primary disadvantage is to be prohibitively uneconomical and impractical to defend possible vulnerability.

\section{COMPARATIVE STUDY OF STANDARD INFORMATION SECURITY RISKS ASSESSMENT FRAMEWORKS}

One of the main conclusions that can be drawn from the study of risk management frameworks is that various frameworks have variations in approach, scope, and applicability. Some methods are designed to be used as stand-alone risk assessment methods, while others are designed to work in conjunction with more general, enterprise-wide risk management processes [12]. Some describe the risk assessment process at a very high granularity and with technical detail, while others simply sketch high-level overviews on how a risk assessment could be undertaken and suggest guidelines or best practices that should be taken into consideration by any such attempt.

Table 2 Comparison of standard risk management frameworks

\begin{tabular}{|c|l|l|l|l|l|l|}
\hline Name & Approach & $\begin{array}{l}\text { Iden } \\
\text { tify } \\
\text { Risk } \\
\text { s? }\end{array}$ & Purpose & $\begin{array}{l}\text { Complex } \\
\text { ity }\end{array}$ & Tool & $\begin{array}{l}\text { Docume } \\
\text { ntation }\end{array}$ \\
\hline FAIR & Yethod & Yes & Academic & Low & $\begin{array}{l}\text { No software tool } \\
\text { defined but has } \\
\text { supporting } \\
\text { documentation }\end{array}$ & Free \\
\hline NIST & Guideline & Yes & $\begin{array}{l}\text { Commerci } \\
\text { al }\end{array}$ & High & Paid Tool & Paid \\
\hline TARA & Guideline & Yes & $\begin{array}{l}\text { Governme } \\
\text { nt }\end{array}$ & Medium & Not defined & Free \\
\hline Mehari & $\begin{array}{l}\text { Method } \\
\text { based }\end{array}$ & Yes & $\begin{array}{l}\text { Commerci } \\
\text { al }\end{array}$ & High & Not defined & $\begin{array}{l}\text { organiz } \\
\text { ational } \\
\text { purpose } \\
\text { only }\end{array}$ \\
\hline
\end{tabular}

There are numerous risk assessment frameworks; however, some of these only provide a guideline while some others propose method abstractly, also, none of them present guideline about the software tools; there is still need of an operational risk management framework that 
DOI: https://dx.doi.org/10.26808/rs.ca.i8v2.08

should precisely define approach, method and implementation details. An operational risk management framework is still needed which could be affordable to small and mid-size organizations.

\section{CONCLUSION}

There are many information security risks assessment frameworks exist today, and it is a tedious task for an organization to choose the proper method. This study aims to compare and analyze the prominent five information security risk assessment frameworks. This analysis will be helpful for the companies, risk experts to find major attributes related to each method. The comparative study finds that the existing information security risks assessment frameworks are method based and involve complex calculations regarding managing risks. Also, the defined process of existing frameworks is complicated to predict. There is no integrated model to assess the security risk quantitatively and optimize its resources to protect organization information and assets effectively; even the best-practice security risk management frameworks do not provide adequate information for effective risk management. Picking risk assessment model without investigation and examination, results in the implementation of security controls in the wrong places, wasting or misusing of resources and leaving an organization vulnerable to unanticipated threats.

For future work, there is need of development of an operation based Information Security Risks Management framework that should be simple and clearly defined the security risks management process. We will focus on development of framework which provides a potential solution to problems of information security management and assessing the probability of incidents and evaluating their expected damages.

\section{ACKNOWLEDGEMENT}

Authors are thankful to Vikram University, Ujjain for providing support and financial grant for the research work.

\section{REFERENCE}

[1] C. Joshi and U. K Singh, "Information security risks management framework - A step towards mitigating security risks in university network", Journal of Information Security and Applications, Elsevier, Vol. 35, pp. 128-137, June 2017.

[2] “Internet Security Threat Report”, Internet Report Volume 21, Apr. 2016.

[3] CVSS Severity Distribution over Time. Available at: https://nvd.nist.gov/vuln-metrics/visualizations/cvss-severity-distribution-over-time

[4] Z. Chen, Y. Zhang, and Z. Chen, "A Categorization Framework for Common Vulnerabilities and Exposures', Computer Journal Advance Access published online on May 7, 2009.

[5] U. K. Singh and C. Joshi, "Information Security Risk Management Framework for University Computing Environment", International Journal of Network Security, Vol.19(5), pp.742-751, Sep. 2017.

[6] C. Alberts, and A. Dorofee, "An Introduction to the OCTAVE Method. Software Engineering Institute", Carnegie Mellon University, USA, 2010.

[7] U. K. Singh and Joshi C, "Information Security Assessment by Quantifying Risk Level of Network Vulnerabilities", International Journal of Computer Application (IJCA 0975 - 8887), Volume 156(2), pp 6-10, December 2016. 
DOI: https://dx.doi.org/10.26808/rs.ca.i8v2.08

[8] B. Dixon, "Understanding the FAIR Risk Assessment", Nebraska CERT Conference 2009.

[9] Guide for Applying the Risk Management Framework to Federal Information Systems, U.S. Department of Commerce, February 2010.

[10] Prioritizing Information Security Risks with Threat Agent Risk Assessment, whitepaper, February 2010.

[11] S. Sinha, Beginning Ethical Hacking with Python, In book: Beginning Ethical Hacking with Python, pp.159-163, Dec. 2017

[12] U. K. Singh and C. Joshi, Quantitative Security Risk Evaluation using CVSS Metrics by Estimation of Frequency and Maturity of Exploit, The World Congress on Engineering and Computer Science (WCECS 2016) San Francisco, USA, Oct. 2016. 